\title{
PCSK9 as a therapeutic target for cardiovascular disease (Review)
}

\author{
PEI-YING ZHANG \\ Department of Cardiology, Xuzhou Central Hospital, The Affiliated Xuzhou Hospital of Medical College \\ of Southeast University, Xuzhou, Jiangsu 221009, P.R. China
}

Received June 16, 2016; Accepted January 12, 2017

DOI: 10.3892/etm.2017.4055

\begin{abstract}
It is well recognized that the elevated plasma level of low-density lipoprotein-cholesterol (LDL-C) is a major risk factor for atherosclerosis and cardiovascular disease (CVD). Deposition of pro-atherogenic LDL-C, on the intima of arterial wall, contributes to plaque formation and atherosclerosis, which further leads to lowered blood flow to vital organs and increased risk of CVD. The most commonly used statin therapy is effective in reducing dyslipidemia and preventing cardiovascular events only in about half of the patient population. However, in patients with familial hypercholesterolemia, these drugs were not effective to meet the required goals of lower LDL-C, and to reduce the CVD risk. Furthermore, many patients even develop intolerability to statins and resistance. The identification of pro-protein convertase subtilisin/kexin type 9 (PCSK9) and the association of PCSK9 mutations with familial hypercholesterolemia led to the identification of PCSK9 as a new therapeutic target for lowering LDL-C and dyslipidemia-associated CVD. PCSK9 is found to promote the degradation of LDL-receptor (LDLR), thus rendering it unavailable for recycling to hepatocyte plasma membrane, leading to elevated levels of circulating LDL-C, as it cannot be taken up into cells. While gain-of-function mutations aggravate the degradation of LDLR as in familial hypercholesterolemia whereas loss of function mutations reduce the ability of PCSK9 to promote the degradation of LDLR and thus lower the plasma level of LDL-C and dyslipidemia. Monoclonal antibodies against PCSK9 are currently being tested in clinical trials and are found to be efficacious in countering the activity of PCSK9 and thus control the plasma LDL-C and triglycerides even in statin non-responsive patients and protect against dyslipidemia-related CVD.
\end{abstract}

Correspondence to: Dr Pei-Ying Zhang, Department of Cardiology, Xuzhou Central Hospital, The Affiliated Xuzhou Hospital of Medical College of Southeast University, 199 South Jiefang Road, Xuzhou, Jiangsu 221009, P.R. China

E-mail: zpying58@126.com

Key words: lipoprotein-cholesterol, atherosclerosis, cardiovascular disease, pro-protein convertase subtilisin/kexin type 9, low-density lipoprotein-receptor, familial hypercholesterolemia, dyslipidemia

\section{Contents}

1. Introduction

2. PCSK9

3. PCSK9 and LDLR degradation

4. PCSK9 mutations and altered lipidemia

5. PCSK9 antagonists as therapeutic agents

6. Conclusions

\section{Introduction}

Cardiovascular disease (CVD) is a major health problem worldwide and despite significant advances in treatment approaches that led to reduced CVD-related mortality over the last few years, the number of individuals living with damaged heart has increased. It is well recognized that elevated plasma level of low-density lipoprotein-cholesterol (LDL-C) is a cardinal risk factor for atherosclerosis and CVD $(1,2)$. Deposition of proatherogenic LDL-C, on the intima of arterial wall, a process mediated by macrophages, contributes to the plaque formation and atherosclerosis. This further leads to lowered blood flow to vital organs and increased risk of atherothrombotic and atheroembolic sequelae. Several clinical studies have shown a direct association between LDL-C reduction and coronary heart disease prevention $(1,3)$. It is known that every $1 \mathrm{mM}$ decrease in plasma LDL-C lowers the risk of cardiovascular events by $20-22 \%(4,5)$. A target reduction of LDL-C to $<2.5 \mathrm{mM}$ for high-risk patients and $<1.8 \mathrm{mM}$ for very high-risk patients has been suggested by the European guidelines for managing dyslipidemia (6). The identification of statins, the inhibitors of 3-hydroxy-3-methylglutaryl-coenzyme A (HMG-CoA) reductase, the rate-limiting enzyme of cholesterol biosynthesis, revolutionized the treatment of dyslipidemia and due to their overwhelming success in reducing hypercholesterolemia and reducing LDL-C they rapidly became the choice of first line therapy for this indication (7). However, statin therapy was effective in reducing dyslipidemia and preventing cardiovascular events only in $50 \%$ of the patient population (4) and in some patients, particularly those with familial hypercholesterolemia, these drugs were not effective to meet the required goals of lower LDL-C, and to reduce the CVD risk (8). In addition, many patients even develop intolerability to statins and resistance (9).

Thus there has been a significant impetus to develop alternate therapeutics to meet the requirements of reducing LDL-C 
and the incidence of cardiovascular events. Several such programs to develop novel therapeutics were not successful due to safety issues and lack of better efficacy over statins $(10,11)$, except ezetimide, which has been found to further improve dyslipidemia over statin therapy (12). Even combination of lipid lowering drugs such as fibrates and nicotinic acid derivatives failed to improve CVD events, when used in conjunction with statins (13). Thus, clinical guidelines do not recommend the use of non-statin drugs in combination with high-intensity statin treatment (14). The finding of pro-protein convertase subtilisin/kexin type 9 (PCSK9) (15) and association of mutations in this protein with familial hypercholesterolemia (16) led to the identification of PCSK9 as a new therapeutic target for lowering LDL-C and dyslipidemia-associated CVD.

\section{PCSK9}

PCSK9 belongs to a family of intracellular 'convertase' or subtilase enzymes that process precursor protein, and are generally inactive, to active mature products that are functional. Substrates for these convertases include a wide array of precursor proteins, such as hormones, receptors, growth factors and other enzymes (17). However, PCSK9 per se does not have an enzymatic function except for the autocatalytic cleavage of its own pro-domain, to become a mature protein. PCSK 9 is encoded by a $22-\mathrm{kb}$ long gene consisting of 12 exons and located at chromosome $1 \mathrm{p} 32$. PCSK 9 gene codes for a 692-amino acid protein of $74 \mathrm{kDa}$ molecular weight, which later undergoes autocatalytic cleavage to the mature $62 \mathrm{kDa}$ form, in endoplasmic reticulum/Golgi bodies, from where it is secreted into circulation. The cleaved prodomain remains non-covalently associated with the mature $62 \mathrm{kDa}$ protein and is essential for the biological function of PCSK9 (18). PCSK9 is primarily synthesized in hepatocytes but other tissues including intestine, brain and kidneys are also known to express this protein $(19,20)$. The transcription factor sterol regulatory element-binding protein 2 (SREBP-2) upregulates PCSK9 expression (21) and LDL-receptor (LDLR) as well as enzymes involved in cholesterol biosynthesis, such as HMG-CoA reductase. Unlike other proconvertase enzymes, PCSK9 is secreted as a complex of mature PCSK9 (153-692 aa) and its inhibitory pro-segment (aa 32-152) $(15,22)$. This complex of PCSK9 is enzymatically inactive as its active site is blocked by the inhibitory pro-segment and thus prevents it from binding with any other substrates (23). Thus, it appears that PCSK9 is its own substrate and its physiological activity probably resides in its ability to bind specific target proteins to escort them towards intracellular degradation compartments.

\section{PCSK9 and LDLR degradation}

The first and probably the most studied and important target of PCSK9 is LDLR on the hepatocyte surface in liver (22). It has been shown in a mouse model that PCSK9 inactivation leads to a significant reduction in cholesteryl esters and atherosclerosis, whereas the overexpression of PCSK9 led to opposite changes and excessive atherosclerosis and all these effects of PCSK9 expression were absent in the LDLR-KO mouse (24). PCSK9 is shown to associate with the epidermal growth factorA (EGF-A) domain of LDLR and to other similar receptors such as the VLDL receptor (25). Previous studies have indicated that PCSK9 is involved in the regulation of plasma triglyceride rich protein and thus it was observed that a deficiency of PCSK9 is associated with significantly lowered plasma triglycerides both in the clinical setting (26) and in genetically altered mice (27). The complex of LDL-C/LDLR normally enters cells through clathrin heavy chain-coated vesicles, followed by its dissociation in the acidic environment of endosomes to LDLR and and LDL-C. LDLR is usually recycled back to the cell surface, whereas the LDL-C is degraded in lysosomes, where the recovered cholesterol is reutilized in the cell (Fig. 1) (28).

However, the complex of LDLR-PCSK9, which also enters the cells through the clathrin-coated vesicles, does not dissociate and is degraded in lysosomes, via some unknown mechanism (29). PCSK9 is shown to direct LDLR for degradation through intracellular pathways 48 and also after its secretion extracellularly by binding to the LDLR (30). While there is ample evidence for these two pathways in hepatocytes, it is not clear if the two pathways are operational in other cells where PCSK9 is expressed. The Cys/His-rich domain present at the C-terminus of PCSK9 appears to be essential for the intracellular and the extracellular LDLR degradation pathways mediated by PCSK9 (31). It has been suggested that the acidic environment of endosomes causes His residues of CHRD domain to be positively charged, making its interaction with the negatively charged ligand-binding domain of LDLR stronger and facilitating its targeting to lysosomes for degradation (Fig. 1) (32). Even though it has been suggested that the amyloid precursor-like protein 2 (APLP-2) participates in the targeting of PCSK9-LDLR complex to lysosomes, through its interaction with CHRD at the surface (33), knockdown of APLP-2 had no effect on PCSK9-mediated LDLR degradation, either via the intracellular or extracellular pathways (18). Interaction of plasma LDL, in particular, the ApoB component of LDL, can bind to circulating PCSK9 and compromise its ability to bind with cell surface LDLR. Thus several players influence the PCSK9-mediated targeting and degradation of LDLR, including protein $\mathrm{X}, \mathrm{ApoB}$, and sec24a, although the mechanistic details of these regulations are not entirely clear. Thus, there is a positive correlation between circulating PCSK9 and LDL-C, as increased levels of PCSK9 lead to LDLR degradation, rendering the cells unable to import LDL-C from circulation. By contrast, binding to LDLR followed by intracellular clearance seems to be the major route for the removal of PCSK9 from circulation, as demonstrated in LDLR-KO mice (34) and in homozygous familial hypercholesterolemia patients (35).

\section{PCSK9 mutations and altered lipidemia}

Following its discovery by Seidah et al (17), mutations in PCSK9 were found to be associated with familial hypercholesterolemia in a French family and a realization that these are gain-of-function mutations $(16,36)$, led to the hypothesis that loss-of-function mutations and inhibition of PCSK9 activity can be beneficial in curtailing dyslipidemia (Fig. 1). Gain-of-function mutations of PCSK9 cause an increased level of this protein in circulation and thus elevated LDL-C. It was also noted that D374Y mutant PCSK9 has a much higher affinity for LDLR as compared with the wild-type 

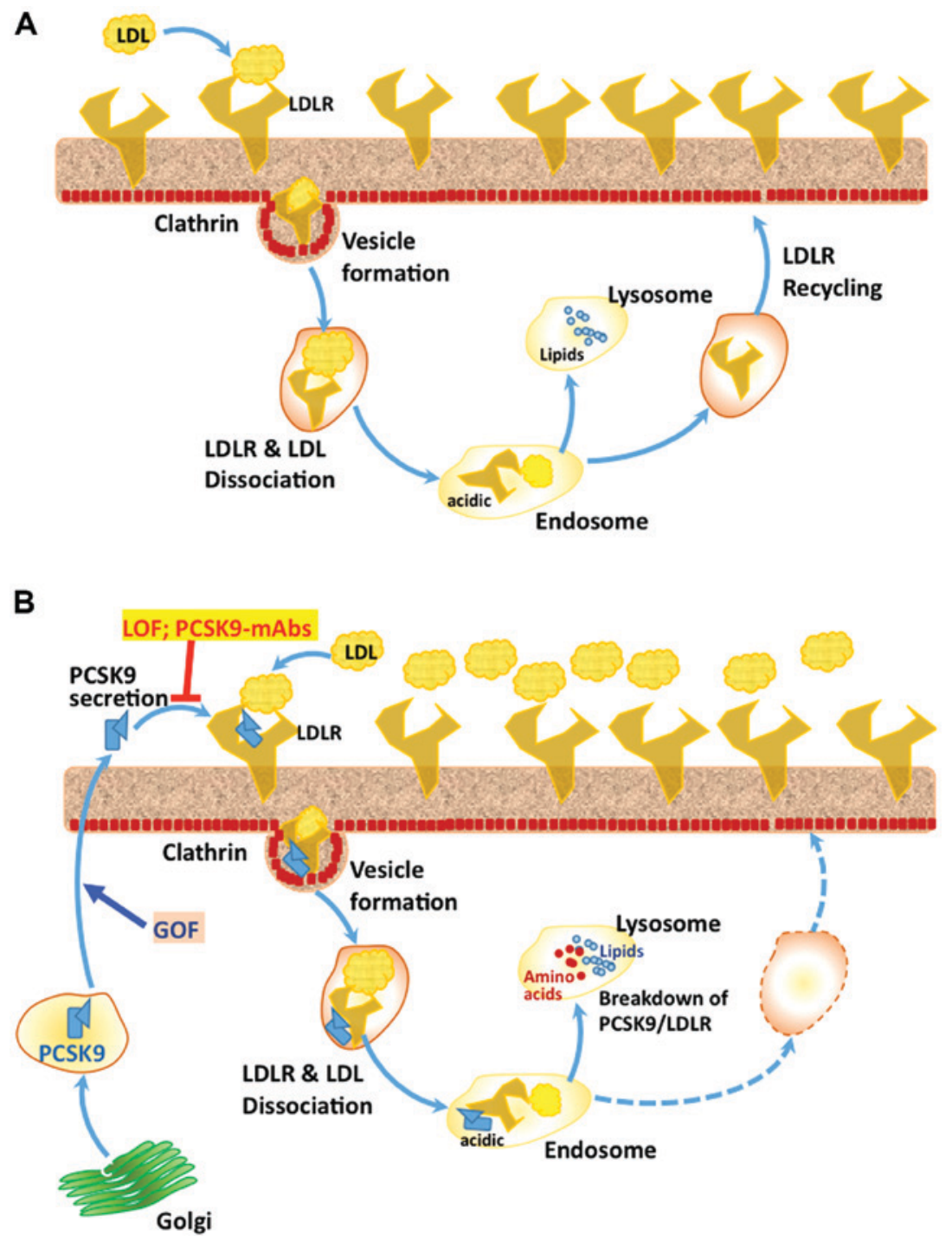

Figure 1. PCSK9-mediated LDLR degradation pathway. (A) In the absence of bound PCSK9, following binding with LDL, LDLR on hepatocyte membrane surface is internalized through clathrin coated vesicles, which form endosomes in cytosol. The acidic environment of the endosome causes dissociation of LDLR and LDL particle, followed by the recycling of LDLR molecules back to the cell surface. However, the LDL particle is taken up by the lysosomes, where complex lipids are broken down to individual components and released into cytosol for further processing and use. (B) In the presence of PCSK9, which associates with LDLR and LDL through different domains, the complex of LDLR-PCSK9-LDL does not dissociate in the acidic environment of endosomes and is degraded into amino acids and individual lipid components, which are released into cytosol. Thus in the presence of PCSK9, there is no recycling of LDLR. Gain of function (GOF) mutations (e.g., D374Y) in PCSK9 increase its affinity for LDLR and augment its overall effect on the breakdown of LDLR. On the other hand, loss-of-function (LOF) mutations in PCSK9 (e.g., Y142X and C679X) and also treatment with therapeutic monoclonal antibodies against PCSK9 result in decreased circulating levels of PCSK9 and thus increased availability of LDLR on the cell surface and significantly lowered plasma LDL-C. PCSK9, pro-protein convertase subtilisin/kexin type 9; LDLR, low-density lipoprotein-receptor; LDL, low-density lipoprotein.

PCSK9 and subjects with this mutation are found to be highly susceptible to develop premature coronary heart disease (23) with severe form of familial hypercholesterolemia and are also less responsive to statins (37). Another gain-of-function mutation, R218S, was found to significantly decrease PCSK9 catabolism, thus promoting its increased circulation time and also high cholesterol levels (38).

Besides the gain-of-function mutations, mutations that led to its loss of function were also identified and were found to be beneficial. Thus, it was observed that carriers of two nonsense PCSK9 variants, Y142X and C679X, decreased plasma LDL-C by $\sim 1.0 \mathrm{mM}$ and the incidence of CVD in these subjects was reduced by $\sim 88 \%$. Similarly, another PCSK 9 variant, R46L, is known to be associated with an LDL-C reduction of $0.5 \mathrm{mM}$, and a $47 \%$ reduction in CVD risk (39).
Protein stability of R46L mutant PCSK9 is reported to be decreased with increased degradation, as well as its affinity for LDLR (40). There is also certain ethnicity-dependent occurrence of a given mutation. Thus, Y142X and C679X mutations are seen in $2 \%$ of African-Americans whereas $\mathrm{R} 46 \mathrm{~L}$ is present in 2-4\% of Caucasian populations (41). R46L mutation is also reported to protect against myocardial infarction (42). Other losses of function PCSK9 mutations are the truncating mutations W428X and L82X (43). In an interesting case of compound heterozygous woman, Y142X mutation of PCSK9 was inherited from the mother and c290-292 delGCC mutation was from the father. The woman had no detectable PCSK9 in circulation with only $0.36 \mathrm{mM}$ LDL-C and lived a healthy normal life, indicating that PCSK9 is not necessary for normal life (44). A specific French-Canadian mutation Q152H, 
which causes loss of function of PCSK9, has been shown to prevent the autocatalytic processing of proPCSK9, leading to the formation of a dominant negative form of PCSK9, which reduces the circulating levels of PCSK9 and also LDL-C by $\sim 50 \%$ (45).

\section{PCSK9 antagonists as therapeutic agents}

Several strategies have been examined to develop PCSK9 antagonists because of the promise they hold as therapeutic agents against dyslipidemia and associated CVD. Attempts to develop small molecule inhibitors, peptidomimetics, were not successful because of specificity and accessibility issues. To date it appears that monoclonal antibodies against PCSK9 are the best approach to block PCSK9 function (Fig. 1), in a therapeutic manner $(18,46)$. Many pharmaceutical companies such as Sanofi (Gentilly, France) and Amgen (Thousand Oaks, CA, USA) have developed different therapeutic monoclonal antibodies against PCSK9 (evolocumab, alirocumab and bococizumab), which have been clinically tested in several trials and found to be safe and efficacious in reducing LDL-C and also triglycerides.

Evolocumab (AMG145), developed by Amgen, is a fully human monoclonal antibody inhibitor of PCSK9 and several meta-analyses recently have shown that this $\mathrm{mAb}$ is well tolerated and more efficacious than statins in patients with familial hypercholesterolemia $(47,48)$. Evolocumab was also found to reduce cardiovascular outcomes in statin treated patients (49). Alirocumab (SAR236553/REGN727), also a fully humanized monoclonal antibody was developed by Sanofi and is found to be almost equipotent as evolocumab in controlling LDL-C, triglycerides and also cardiovascular outcomes (49).

Clinical experience with the anti-PCSK9-monoclonal antibodies indicates that these therapeutic agents are safe and well tolerated, without any major safety issues and serious drug-related adverse events (50). The most common adverse events were nasopharyngitis, upper respiratory tract infections, influenza-like symptoms and back pain (51). Even though treatment related adverse effects were not noted in patients receiving PCSK9 $\mathrm{mAbs}$, caution needs to be exercised for possible hypocholesterolemia associated adverse effects such as cognitive impairment as well as hemorrhagic stroke (49). More clinical data are needed to ascertain if these are potential problems or of not much concern.

\section{Conclusions}

Plasma level of LDL-C is a major risk factor for atherosclerosis and CVD. The most commonly used statin therapy is effective in reducing dyslipidemia and preventing cardiovascular events only in about half of the patient population and these drugs are not effective in patients with familial hypercholesterolemia, to meet the required goals of lower LDL-C, to reduce the CVD risk. The finding of PCSK9, which promotes LDLR breakdown and association of PCSK9 gain of mutations with familial hypercholesterolemia and loss of mutations with reduced levels of LDL-C, led to the identification of PCSK9 as a new therapeutic target for lowering LDL-C and dyslipidemia associated CVD. Monoclonal antibodies against PCSK9 are currently being tested in clinical trials and have been found to be safe without any major adverse effects and efficacious in countering the activity of PCSK9 and thus control the plasma LDL-C and triglycerides even in statin-non responsive patients and protect against dyslipidemia-related CVD. Further research is needed to clearly establish their protective effect against CVD and related mortality.

\section{References}

1. Law MR, Wald NJ and Rudnicka AR: Quantifying effect of statins on low density lipoprotein cholesterol, ischaemic heart disease, and stroke: systematic review and meta-analysis. BMJ 326: 1423, 2003.

2. Mendis S, Puska P and Norrving B: Global atlas on cardiovascular disease prevention and control. World Health Organization, Geneva, 2011

3. Brown MS and Goldstein JL: Biomedicine. Lowering LDL - not only how low, but how long? Science 311: 1721-1723, 2006.

4. Mihaylova B, Emberson J, Blackwell L, Keech A, Simes J, Barnes EH, Voysey M, Gray A, Collins R and Baigent C; Cholesterol Treatment Trialists' (CTT) Collaborators: the effects of lowering LDL cholesterol with statin therapy in people at low risk of vascular disease: meta-analysis of individual data from 27 randomised trials. Lancet 380: 581-590, 2012.

5. Baigent C, Blackwell L, Emberson J, Holland LE, Reith C, Bhala N, Peto R, Barnes EH, Keech A, Simes J, et al; Cholesterol Treatment Trialists' (CTT) Collaboration: Efficacy and safety of more intensive lowering of LDL cholesterol: a meta-analysis of data from 170,000 participants in 26 randomised trials. Lancet 376: 1670-1681, 2010.

6. Reiner Z, Catapano AL, De Backer G, Graham I, Taskinen MR, Wiklund O, Agewall S, Alegria E, Chapman MJ, Durrington P, et al; European Association for Cardiovascular Prevention \& Rehabilitation; ESC Committee for Practice Guidelines (CPG) 2008-2010 and 2010-2012 Committees: ESC/EAS Guidelines for the management of dyslipidaemias: The Task Force for the management of dyslipidaemias of the European Society of Cardiology (ESC) and the European Atherosclerosis Society (EAS). Eur Heart J 32: 1769-1818, 2011.

7. Koo BK: Statin for the primary prevention of cardiovascular disease in patients with diabetes mellitus. Diabetes Metab J 38: 32-34, 2014.

8. Joy TR: Novel therapeutic agents for lowering low density lipoprotein cholesterol. Pharmacol Ther 135: 31-43, 2012.

9. Pijlman AH, Huijgen R, Verhagen SN, Imholz BP, Liem AH, Kastelein JJ, Abbink EJ, Stalenhoef AF and Visseren FL: Evaluation of cholesterol lowering treatment of patients with familial hypercholesterolemia: a large cross-sectional study in The Netherlands. Atherosclerosis 209: 189-194, 2010.

10. Barter PJ, Caulfield M, Eriksson M, Grundy SM, Kastelein JJ, Komajda M, Lopez-Sendon J, Mosca L, Tardif JC, Waters DD, et al; ILLUMINATE Investigators: Effects of torcetrapib in patients at high risk for coronary events. N Engl J Med 357: 2109-2122, 2007.

11. Landray MJ, Haynes R, Hopewell JC, Parish S, Aung T, Tomson J, Wallendszus K, Craig M, Jiang L, Collins R, et al; HPS2-THRIVE Collaborative Group: Effects of extended-release niacin with laropiprant in high-risk patients. N Engl J Med 371: 203-212, 2014.

12. Cannon CP, Blazing MA, Giugliano RP, McCagg A, White JA, Theroux P, Darius H, Lewis BS, Ophuis TO, Jukema JW, et al; IMPROVE-IT Investigators: Ezetimibe added to statin therapy after acute coronary syndromes. N Engl J Med 372: 2387-2397, 2015

13. Ginsberg HN, Elam MB, Lovato LC, Crouse JR III, Leiter LA, Linz P, Friedewald WT, Buse JB, Gerstein HC, Probstfield J, et al; ACCORD Study Group: Effects of combination lipid therapy in type 2 diabetes mellitus. N Engl J Med 362: 1563-1574, 2010.

14. Stone NJ, Robinson JG, Lichtenstein AH, Bairey Merz CN, Blum CB, Eckel RH, Goldberg AC, Gordon D, Levy D, Lloyd-Jones DM, etal; American College of Cardiology/American Heart Association Task Force on Practice Guidelines: 2013 ACC/AHA guideline on the treatment of blood cholesterol to reduce atherosclerotic cardiovascular risk in adults: a report of the American College of Cardiology/American Heart Association Task Force on Practice Guidelines. J Am Coll Cardiol 63: 2889-2934, 2014. 
15. Seidah NG, Benjannet S, Wickham L, Marcinkiewicz J, Jasmin SB, Stifani S, Basak A, Prat A and Chretien M: The secretory proprotein convertase neural apoptosis-regulated convertase 1 (NARC-1): liver regeneration and neuronal differentiation. Proc Natl Acad Sci USA 100: 928-933, 2003.

16. Abifadel M, Varret M, Rabès JP, Allard D, Ouguerram K, Devillers M, Cruaud C, Benjannet S, Wickham L, Erlich D, et al: Mutations in PCSK9 cause autosomal dominant hypercholesterolemia. Nat Genet 34: 154-156, 2003.

17. Seidah NG, Sadr MS, Chrétien M and Mbikay M: The multifaceted proprotein convertases: their unique, redundant, complementary, and opposite functions. J Biol Chem 288: 21473-21481, 2013.

18. Seidah NG, Awan Z, Chrétien M and Mbikay M: PCSK9: a key modulator of cardiovascular health. Circ Res 114: 1022-1036, 2014.

19. Artenstein AW and Opal SM: Proprotein convertases in health and disease. N Engl J Med 365: 2507-2518, 2011

20. Seidah NG and Prat A: The biology and therapeutic targeting of the proprotein convertases. Nat Rev Drug Discov 11: 367-383, 2012

21. Jeong HJ, Lee HS, Kim KS, Kim YK, Yoon D and Park SW: Sterol-dependent regulation of proprotein convertase subtilisin/kexin type 9 expression by sterol-regulatory element binding protein-2. J Lipid Res 49: 399-409, 2008.

22. Benjannet S, Rhainds D, Essalmani R, Mayne J, Wickham L, Jin W, Asselin MC, Hamelin J, Varret M, Allard D, et al: NARC-1/PCSK9 and its natural mutants: Zymogen cleavage and effects on the low density lipoprotein (LDL) receptor and LDL cholesterol. J Biol Chem 279: 48865-48875, 2004.

23. Cunningham D, Danley DE, Geoghegan KF, Griffor MC, Hawkins JL, Subashi TA, Varghese AH, Ammirati MJ, Culp JS, Hoth LR, et al: Structural and biophysical studies of PCSK9 and its mutants linked to familial hypercholesterolemia. Nat Struct Mol Biol 14: 413-419, 2007.

24. Denis M, Marcinkiewicz J, Zaid A, Gauthier D, Poirier S, Lazure C, Seidah NG and Prat A: Gene inactivation of proprotein convertase subtilisin/kexin type 9 reduces atherosclerosis in mice. Circulation 125: 894-901, 2012.

25. Lo Surdo P, Bottomley MJ, Calzetta A, Settembre EC, Cirillo A, Pandit S, Ni YG, Hubbard B, Sitlani A and Carfí A Mechanistic implications for LDL receptor degradation from the PCSK9/LDLR structure at neutral pH. EMBO Rep 12: $1300-1305,2011$

26. Akram ON, Bernier A, Petrides F, Wong G and Lambert G: Beyond LDL cholesterol, a new role for PCSK9. Arterioscler Thromb Vasc Biol 30: 1279-1281, 2010.

27. Le May C, Kourimate S, Langhi C, Chétiveaux M, Jarry A, Comera C, Collet X, Kuipers F, Krempf M, Cariou B, et al: Proprotein convertase subtilisin kexin type 9 null mice are protected from postprandial triglyceridemia. Arterioscler Thromb Vasc Biol 29: 684-690, 2009.

28. Huang S, Henry L, Ho YK, Pownall HJ and Rudenko G: Mechanism of LDL binding and release probed by structure-based mutagenesis of the LDL receptor. J Lipid Res 51: 297-308, 2010.

29. Nassoury N, Blasiole DA, Tebon Oler A, Benjannet S, Hamelin J, Poupon V, McPherson PS, Attie AD, Prat A and Seidah NG: The cellular trafficking of the secretory proprotein convertase PCSK9 and its dependence on the LDLR. Traffic 8: 718-732, 2007.

30. Cameron J, Holla OL, Ranheim T, Kulseth MA, Berge KE and Leren TP: Effect of mutations in the PCSK9 gene on the cell surface LDL receptors. Hum Mol Genet 15: 1551-1558, 2006.

31. Saavedra YG, Day R and Seidah NG: The M2 module of the Cys-His-rich domain (CHRD) of PCSK9 protein is needed for the extracellular low-density lipoprotein receptor (LDLR) degradation pathway. J Biol Chem 287: 43492-43501, 2012.

32. Tveten K, Holla OL, Cameron J, Strøm TB, Berge KE, Laerdahl JK and Leren TP: Interaction between the ligand-binding domain of the LDL receptor and the C-terminal domain of PCSK9 is required for PCSK9 to remain bound to the LDL receptor during endosomal acidification. Hum Mol Genet 21: 1402-1409, 2012.

33. DeVay RM, Shelton DL and Liang H: Characterization of proprotein convertase subtilisin/kexin type 9 (PCSK9) trafficking reveals a novel lysosomal targeting mechanism via amyloid precursor-like protein 2 (APLP2). J Biol Chem 288 : 10805-10818, 2013.

34. Tavori H, Fan D, Blakemore JL, Yancey PG, Ding L, Linton MF and Fazio S: Serum proprotein convertase subtilisin/kexin type 9 and cell surface low-density lipoprotein receptor: Evidence for a reciprocal regulation. Circulation 127: 2403-2413, 2013.
35. Raal F, Scott R, Somaratne R, Bridges I, Li G, Wasserman SM and Stein EA: Low-density lipoprotein cholesterol-lowering effects of AMG 145, a monoclonal antibody to proprotein convertase subtilisin/kexin type 9 serine protease in patients with heterozygous familial hypercholesterolemia: The Reduction of LDL-C with PCSK9 Inhibition in Heterozygous Familial Hypercholesterolemia Disorder (RUTHERFORD) randomized trial. Circulation 126: 2408-2417, 2012.

36. Leren TP: Mutations in the PCSK9 gene in Norwegian subjects with autosomal dominant hypercholesterolemia. Clin Genet 65: 419-422, 2004.

37. Stefanutti C, Morozzi C and Di Giacomo S: New clinical perspectives of hypolipidemic drug therapy in severe hypercholesterolemia. Curr Med Chem 19: 4861-4868, 2012.

38. Allard D, Amsellem S, Abifadel M, Trillard M, Devillers M, Luc G, Krempf M, Reznik Y, Girardet JP, Fredenrich A, et al: Novel mutations of the PCSK9 gene cause variable phenotype of autosomal dominant hypercholesterolemia. Hum Mutat 26: 497, 2005

39. Cohen JC, Boerwinkle E, Mosley TH Jr and Hobbs HH: Sequence variations in PCSK9, low LDL, and protection against coronary heart disease. N Engl J Med 354: 1264-1272, 2006.

40. Dewpura T, Raymond A, Hamelin J, Seidah NG, Mbikay M, Chrétien M and Mayne J: PCSK9 is phosphorylated by a Golgi casein kinase-like kinase ex vivo and circulates as a phosphoprotein in humans. FEBS J 275: 3480-3493, 2008.

41. Benn M, Nordestgaard BG, Grande P, Schnohr P and Tybjaerg-Hansen A: PCSK9 R46L, low-density lipoprotein cholesterol levels, and risk of ischemic heart disease: 3 independent studies and meta-analyses. J Am Coll Cardiol 55: 2833-2842, 2010

42. Kathiresan S; Myocardial Infarction Genetics Consortium: A PCSK9 missense variant associated with a reduced risk of early-onset myocardial infarction. N Engl J Med 358: 2299-2300, 2008.

43. Marais AD, Kim JB, Wasserman SM and Lambert G: PCSK9 inhibition in LDL cholesterol reduction: Genetics and therapeutic implications of very low plasma lipoprotein levels. Pharmacol Ther 145: 58-66, 2015.

44. Zhao Z, Tuakli-Wosornu Y, Lagace TA, Kinch L, Grishin NV, Horton JD, Cohen JC and Hobbs HH: Molecular characterization of loss-of-function mutations in PCSK9 and identification of a compound heterozygote. Am J Hum Genet 79: 514-523, 2006.

45. Mayne J, Dewpura T, Raymond A, Bernier L, Cousins M, Ooi TC, Davignon J, Seidah NG, Mbikay M and Chrétien M: Novel loss-of-function PCSK9 variant is associated with low plasma LDL cholesterol in a French-Canadian family and with impaired processing and secretion in cell culture. Clin Chem 57: 1415-1423, 2011

46. Druce I, Abujrad H and Ooi TC: PCSK9 and triglyceride-rich lipoprotein metabolism. J Biomed Res 29: 29, 2015.

47. Giugliano RP, Desai NR, Kohli P, Rogers WJ, Somaratne R, Huang F, Liu T, Mohanavelu S, Hoffman EB, McDonald ST, et al; LAPLACE-TIMI 57 Investigators: Efficacy, safety, and tolerability of a monoclonal antibody to proprotein convertase subtilisin/kexin type 9 in combination with a statin in patients with hypercholesterolaemia (LAPLACE-TIMI 57): a randomised, placebo-controlled, dose-ranging, phase 2 study. Lancet 380: 2007-2017, 2012

48. Raal FJ, Stein EA, Dufour R, Turner T, Civeira F, Burgess L, Langslet G, Scott R, Olsson AG, Sullivan D, et al; RUTHERFORD-2 Investigators: PCSK9 inhibition with evolocumab (AMG 145) in heterozygous familial hypercholesterolaemia (RUTHERFORD-2): a randomised, double-blind, placebo-controlled trial. Lancet 385: 331-340, 2015.

49. Latimer J, Batty JA, Neely RD and Kunadian V: PCSK9 inhibitors in the prevention of cardiovascular disease. J Thromb Thrombolysis: Apr 19, 2016 (Epub ahead of print).

50. Cicero AF, Tartagni E and Ertek S: Safety and tolerability of injectable lipid-lowering drugs: a review of available clinical data. Expert Opin Drug Saf 13: 1023-1030, 2014.

51. Roth EM, Taskinen MR, Ginsberg HN, Kastelein JJ, Colhoun HM, Robinson JG, Merlet L, Pordy R and Baccara-Dinet MT: Monotherapy with the PCSK9 inhibitor alirocumab versus ezetimibe in patients with hypercholesterolemia: results of a 24 week, double-blind, randomized phase 3 trial. Int J Cardiol 176: 55-61, 2014. 